\title{
Tratamento de efluentes de carcinicultura por macrófitas aquáticas flutuantes
}

\author{
Gustavo Gonzaga Henry-Silva ${ }^{1}$, Antonio Fernando Monteiro Camargo $^{2}$
}

\footnotetext{
${ }^{1}$ Depto. de Ciências Animais - UFERSA/CAUNESP - Mossoró, RN.

${ }^{2}$ Depto. de Ecologia - UNESP/CAUNESP - Rio Claro, SP.
}

\begin{abstract}
RESUMO - Avaliou-se neste estudo a eficiência de sistemas compostos de duas espécies de macrófitas aquáticas flutuantes (Eichhornia crassipes e Pistia stratiotes) no tratamento de efluentes gerados por um viveiro de manutenção de reprodutores de camarões-canela (Macrobrachium amazonicum). Os sistemas de tratamento foram constituídos de 12 unidades experimentais com as macrófitas aquáticas e três sem plantas (controle). Foram analisadas amostras da água de abastecimento do viveiro de criação de camarões-d'água-doce, do efluente gerado e dos efluentes tratados. A remoção de nutrientes não diferiu significativamente entre as duas espécies vegetais. As maiores remoções foram obtidas para fósforo total (41,9\% pelo controle; $71,6 \%$ por E. crassipes; $69,9 \%$ por P. stratiotes; $72,5 \%$ por E. crassipes $+P$. stratiotes e $72,1 \%$ por $P$. stratiotes + E. crassipes) e para turbidez $(30,6 \%$ pelo controle; $80,2 \%$ por E. crassipes; $75,2 \%$ por $P$. stratiotes; $79,8 \%$ por E. crassipes $+P$. stratiotes e $81,5 \%$ por $P$. stratiotes $+E$. crassipes). As macrófitas aquáticas foram eficientes na remoção de nitrogênio e fósforo dos efluentes de criação de M. amazonicum.
\end{abstract}

Palavras-chave: aqüicultura, Eichhornia crassipes, Macrobrachium amazonicum, nutrientes, Pistia stratiotes

\section{Treatment of shrimp effluents by free-floating aquatic macrophytes}

\begin{abstract}
The efficiency of systems composed of two species of floating aquatic macrophyte (Eichhornia crassipes and Pistia stratiotes) to treat effluents from shrimp culture was evaluated in this research. The effluent originated from a pond populated with Macrobrachium amazonicum. Treatment systems consisted of 12 experimental tanks with aquatic macrophyte and three tanks without plants (control). Water samples were collected from the fresh water supply and before and after passing through the treatment systems. There are no differences in the removal of nutrients between the two species. The higher nutrient removal was observed for total phosphorus $(41.9 \%$ by control; $71.6 \%$ by E. crassipes; 69.9 by P. stratiotes; $72.5 \%$ by E. crassipes + P. stratiotes and 72.1 by P. stratiotes + E. crassipes) and turbidity ( $30.6 \%$ by control; $80.2 \%$ by E. crassipes; 75.2 by P. stratiotes; $79.8 \%$ by E. crassipes $+P$. stratiotes and 81.5 by P. stratiotes + E. crassipes) The systems containing aquatic macrophytes were efficient in nitrogen and phosphorus removal from M. amazonicum culture.
\end{abstract}

Key Words: aquaculture, Eichhornia crassipes, Macrobrachium amazonicum, nutrients, Pistia stratiotes

\section{Introdução}

A aqüicultura moderna deve contemplar, além do lucro, a preservação ambiental e o desenvolvimento social. A preocupação com o ambiente deve ser parte integrante do processo de produção, de modo que as técnicas devem otimizar a produção de organismos aquáticos considerando os possíveis impactos do sistema de criação (Valenti, 2000). A aqüicultura, assim como toda a atividade produtiva, acarreta impactos ambientais, especialmente pelos efluentes gerados. Assim, é necessário considerar mecanismos viáveis para minimizar esses impactos, planejando adequadamente a utilização dos recursos naturais e elaborando estratégias eficientes de desenvolvimento susten- tável (Assad \& Bursztyn, 2000; Henry-Silva, 2005). Neste contexto, estratégias apropriadas de manejo dos resíduos são indispensáveis para manter a legalidade, a rentabilidade e a sustentabilidade de qualquer empreendimento aqüícola (NACA/FAO, 2000).

Entre as atividades da aqüicultura, a carcinicultura destaca-se por sua ampla difusão em várias partes do mundo (Cavalcanti, 1998; Ostrensky et al., 2000). Essa atividade desperta a atenção não somente pelo seu potencial em gerar alimento para o homem, mas também por gerar impactos ambientais (Beardmore et al., 1997; Midlen \& Redding, 1998). De modo geral, a alta produção fitoplanctônica nos viveiros de criação de camarões força os carcinicultores a contínuas renovações de água, gerando descargas de 
efluentes nos ambientes aquáticos naturais (Wang, 1990). É importante destacar que as características desses efluentes tendem a apresentar ampla variação, em decorrência das formas de manejo, da tecnologia empregada, das espécies criadas e, principalmente, da intensidade de produção (Castagnolli, 1992; Boyd, 2003). Os principais impactos dos efluentes sobre os ambientes naturais são o aumento das concentrações de nutrientes na água e no sedimento e o incremento das populações de fitoplâncton e de bactérias (Redding et al., 1997). A eutrofização artificial também pode comprometer os sistemas de criação, em decorrência da redução dos teores de oxigênio dissolvido, do aumento de compostos nitrogenados tóxicos e do comprometimento da qualidade do pescado (Pilarski et al., 2004; True et al., 2004; Baccarin \& Camargo, 2005; HenrySilva et al., 2006).

Os sistemas de tratamento de efluentes por meio de macrófitas aquáticas podem ser viáveis para os aqüicultores e ainda minimizam os impactos sobre os ecossistemas aquáticos. Apesar de diversos trabalhos comprovarem a eficiência das plantas aquáticas no tratamento de efluentes domésticos (Ennabili et al., 1998; Lin et al., 2005; Greenway, 2005; Hadad et al., 2006), estudos sobre a utilização desses vegetais no tratamento de efluentes de aqüicultura são recentes no Brasil (Zaniboni-Filho 1997; Sipauba Tavares 2002; Henry-Silva \& Camargo, 2006). Neste trabalho, estudou-se a eficiência das macrófitas aquáticas flutuantes Eichhornia crassipes e Pistia stratiotes no tratamento de efluentes de camarões-canela (Macrobrachium amazonicum).

\section{Material e Métodos}

$\mathrm{O}$ experimento foi desenvolvido na Universidade Estadual Paulista $\left(21^{\circ} 18^{\prime} \mathrm{S}\right.$ e $\left.48^{\circ} 18^{\prime} \mathrm{W}\right)$, no Setor de Carcinicultura do Centro de Aqüicultura, durante o período de janeiro a maio de 2003.

Os sistemas de tratamento dos efluentes foram constituídos de 15 tanques experimentais de fibra de vidro, instalados ao ar livre, e com as seguintes dimensões: 1,0 $\mathrm{m}$ de largura, 2, $0 \mathrm{~m}$ de comprimento e $0,5 \mathrm{~m}$ de profundidade. $\mathrm{O}$ período de residência do efluente em cada um dos tanques foi de aproximadamente 17 horas, com vazão de $1 \mathrm{~L}$ /minuto. O delineamento experimental foi inteiramente casualizado, com cinco tratamentos, cada um com três repetições. Os tratamentos foram compostos de: apenas E. crassipes (3.500 $\left.\mathrm{g} / \mathrm{m}^{2}\right)$; apenas P. stratiotes $\left(2.750 \mathrm{~g} / \mathrm{m}^{2}\right) ; \mathrm{E}+\mathrm{P}$ : E. crassipes na metade inicial dos tanques $\left(1.750 \mathrm{~g} / \mathrm{m}^{2}\right)$ e P. stratiotes na metade final dos tanques $\left(1.375 \mathrm{~g} / \mathrm{m}^{2}\right)$;
P + E: P. stratiotes na metade inicial dos tanques $\left(1.375 \mathrm{~g} / \mathrm{m}^{2}\right)$ e E. crassipes na metade final dos tanques $\left(1.750 \mathrm{~g} / \mathrm{m}^{2}\right)$; controle: sem plantas (Figura 1).

As macrófitas aquáticas utilizadas nas unidades experimentais foram coletadas em ecossistemas lóticos bem preservados do litoral sul do estado de São Paulo ( $24^{\circ} 11^{\prime}$ $\left.\mathrm{S} \mathrm{e} 46^{\circ} 48^{\prime} \mathrm{W}\right)$. A biomassa das espécies vegetais preencheu aproximadamente $80 \%$ da superfície das unidades experimentais. Os efluentes foram provenientes de um viveiro de $200 \mathrm{~m}^{2}$ com aproximadamente 2.000 reprodutores de camarão-canela ( $M$. amazonicum) e densidade média de estocagem de $85,7 \mathrm{~g} / \mathrm{m}^{2}$. O manejo alimentar foi feito duas vezes ao dia com o fornecimento de ração peletizada comercial contendo $32 \%$ de PB, $7 \%$ de EE, $9 \%$ de fibras e $12 \%$ de matéria mineral.

Semanalmente, entre $9 \mathrm{~h}$ e $10 \mathrm{~h}$, foram coletadas e analisadas amostras de água dos viveiros de camarão, dos efluentes gerados, dos efluentes tratados pelas macrófitas aquáticas e dos tanques controle. As medidas de temperatura, potencial hidrogeniônico $(\mathrm{pH})$, turbidez, oxigênio dissolvido e condutividade elétrica foram obtidas utilizando-se o aparelho Water Quality Checker, modelo U-10 da marca Horiba. As determinações de nitrogênio total (NT), $\mathrm{N}$-nitrito $\left(\mathrm{N}-\mathrm{NO}_{2}\right)$ e $\mathrm{N}$-nitrato $\left(\mathrm{N}-\mathrm{NO}_{3}\right)$ foram realizadas segundo método descrito por Mackereth et al. (1978) e as concentrações de $\mathrm{N}$-amoniacal $\left(\mathrm{N}-\mathrm{NH}_{3}\right)$ foram determinadas de acordo com o método proposto por Koroleff (1976). Os valores de fósforo total (PT), fósforo dissolvido (PD) e alcalinidade total foram calculados utilizando-se o método descrito por Golterman et al. (1978).

Semanalmente, as plantas foram removidas das partes inicial (entrada do efluente) e final (saída do efluente tratado) dos tanques para determinação da massa fresca e, posteriormente foram devolvidas às suas respectivas unidades experimentais. Antes da pesagem, o excesso de água retido nas raízes dos vegetais foi retirado, deixando-se a água escorrer por aproximadamente 5 minutos.

A massa seca das macrófitas aquáticas foi estimada pela equação de regressão linear entre a massa fresca (MF) e a massa seca (MS) de indivíduos coletados nos ecossistemas lóticos do litoral sul do estado de São Paulo:

E. crassipes: $\mathrm{MS}=0,565+0,061 \times \mathrm{MF}(\mathrm{r}=0,985 ; \mathrm{n}=30)$;

P. stratiotes: $\mathrm{MS}=-1,951+0,055 \times \mathrm{MF}(\mathrm{r}=0,992 ; \mathrm{n}=30)$.

As porcentagens de remoção dos nutrientes e da turbidez, para cada tratamento, foram calculadas pela equação:

$$
\mathrm{R}=100-\left[\left(100 \times \mathrm{C}_{\text {etrat }}\right) / \mathrm{C}_{\mathrm{e}}\right]
$$

em que: $\mathrm{R}=$ porcentagem de remoção; $\mathrm{C}_{\text {etrat }}=$ concentração de nutriente no efluente tratado e $\mathrm{C}_{\mathrm{e}}=$ concentração de nutriente no efluente de carcinicultura. 


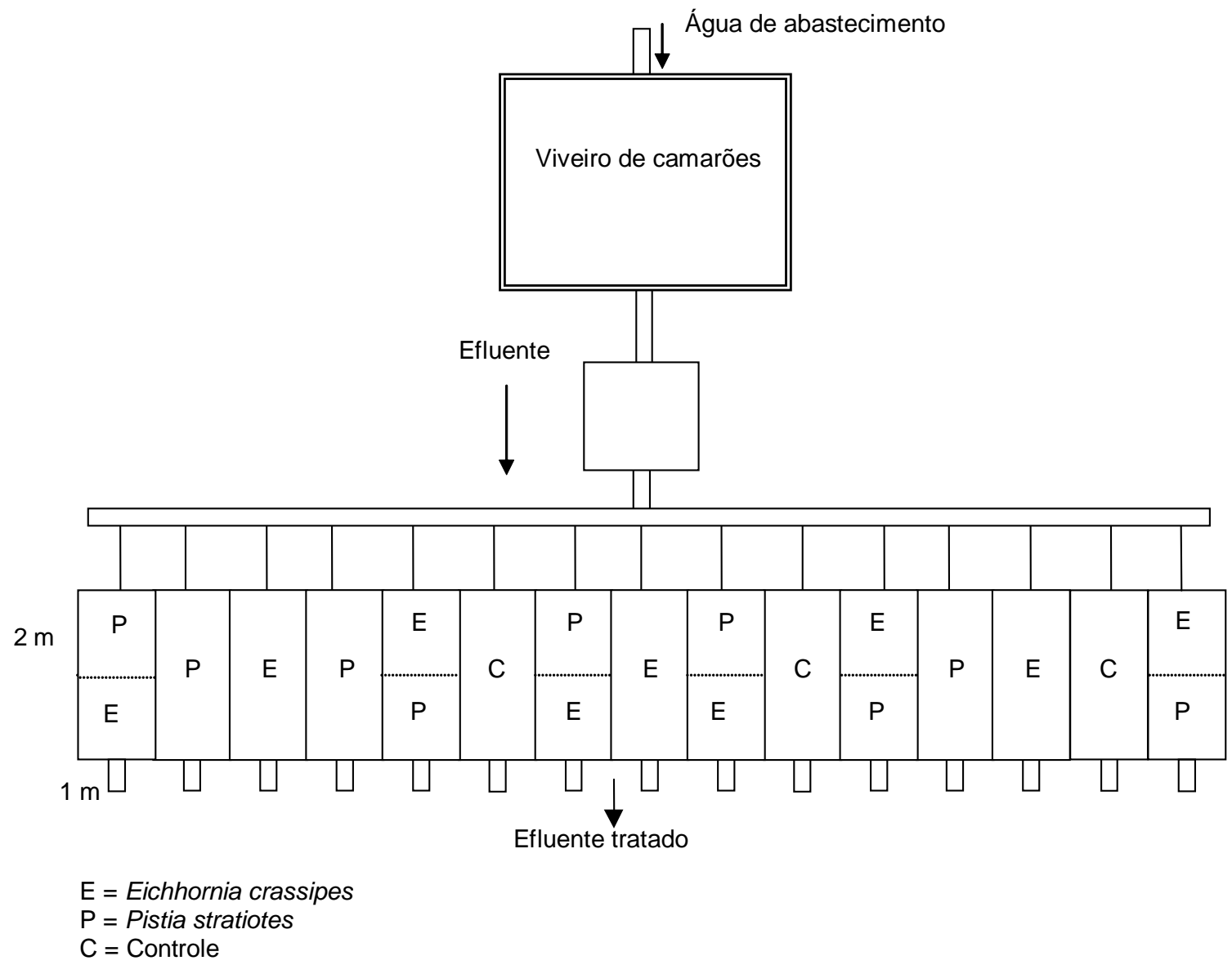

Figura 1 - Diagrama esquemático dos sistemas de tratamento com as macrófitas aquáticas.

Os resultados das variáveis limnológicas do efluente tratado pelas macrófitas foram submetidos à análise de variância (ANOVA one-way) e as médias, quando diferentes, foram comparadas pelo teste Tukey $(\mathrm{P}<0,05)$.

\section{Resultados e Discussão}

Os efluentes dos viveiros apresentaram maiores valores médios para as características físicas e químicas, temperatura, $\mathrm{pH}$, condutividade elétrica, alcalinidade, turbidez, fósforo e nitrogênio em comparação à água de abastecimento. Os aumentos nos valores de turbidez, fósforo total e nitrogênio total no efluente foram de 106,7; 78,4 e 56,7\%, respectivamente. Para as formas nitrogenadas, os incrementos foram de 59,7\% para N-nitrato; $48,0 \%$ para $\mathrm{N}$-nitrito e $50,4 \%$ para $\mathrm{N}$-amoniacal. A concentração de oxigênio dissolvido do efluente reduziu $27,4 \%$ em relação à concentração média obtida na água de abastecimento do viveiro (Tabela 1$)$.

Aumento na concentração de nutrientes e redução dos teores de oxigênio dissolvido também foram observados por Henry-Silva (2001) em efluentes de viveiro de cultivo de tilápia-do-nilo (O. niloticus). O maior valor de temperatura no efluente de carcinicultura pode estar relacionado ao aquecimento pelos raios solares incidentes sobre o viveiro de criação. A temperatura mais elevada também contribuiu para a redução das concentrações de oxigênio dissolvido, em decorrência da redução da solubilização deste gás. O pH

Tabela 1 - Características limnológicas da água de abastecimento e do efluente de criação de Macrobrachium amazonicum

\begin{tabular}{lrr}
\hline Item & Abastecimento & \multicolumn{1}{c}{ Efluente } \\
\hline Temperatura $\left({ }^{\circ} \mathrm{C}\right)$ & $25,7 \pm 2,0$ & $26,5 \pm 2,2$ \\
$\mathrm{pH}$ & $7,5 \pm 0,3$ & $8,1 \pm 0,5$ \\
Oxigênio dissolvido $(\mathrm{mg} / \mathrm{L})$ & $6,5 \pm 0,9$ & $5,1 \pm 0,6$ \\
Condutividade $(\mathrm{mS} / \mathrm{cm})$ & $0,058 \pm 0,008$ & $0,068 \pm 0,006$ \\
Turbidez $(\mathrm{NTU})$ & $30 \pm 7,0$ & $62 \pm 21$ \\
Fósforo total $(\mu \mathrm{g} / \mathrm{L})$ & $128,5 \pm 27,9$ & $229,3 \pm 69,7$ \\
Fósforo dissolvido $(\mu \mathrm{g} / \mathrm{L})$ & $15,6 \pm 5,4$ & $33,1 \pm 7,1$ \\
Nitrogênio total $(\mathrm{mg} / \mathrm{L})$ & $0,30 \pm 0,03$ & $0,47 \pm 0,15$ \\
N-nitrato $(\mu \mathrm{g} / \mathrm{L})$ & $99,1 \pm 12,3$ & $158,3 \pm 23,4$ \\
N-nitrito $(\mu \mathrm{g} / \mathrm{L})$ & $10,0 \pm 1,1$ & $14,8 \pm 2,2$ \\
N-amoniacal $(\mu \mathrm{g} / \mathrm{L})$ & $11,3 \pm 0,9$ & $17,0 \pm 1,4$ \\
\hline
\end{tabular}


mais elevado no efluente provavelmente esteve relacionado à fotossíntese pelo fitoplâncton, que, ao assimilar o $\mathrm{CO}_{2}$ disponível na água, aumentou os valores de $\mathrm{pH}$ do meio (Henry-Silva \& Camargo, 2000).

A qualidade do efluente de carcinicultura (teores de nutrientes e matéria orgânica) melhorou depois que a água passou pelos sistemas de tratamento. Os valores de fósforo, nitrogênio e turbidez do efluente reduziram após o efluente atravessar os sistemas com as macrófitas aquáticas. Os valores obtidos para estas variáveis foram superiores no efluente tratado pelo sistema controle (sem plantas). Não foram constatadas diferenças significativas $(P>0,05)$ entre as características limnológicas do efluente tratado pelos sistemas com E. crassipes; P. stratiotes; E. crassipes $+P$. stratiotes; e P. stratiotes + E. crassipes. Os valores de temperatura, $\mathrm{pH}$, oxigênio dissolvido e condutividade elétrica no efluente tratado pelas macrófitas aquáticas foram menores que os obtidos no efluente da carcinicultura (Tabela 2).

Os menores valores de temperatura no efluente tratado pelas macrófitas aquáticas foram ocasionados principalmente pelo sombreamento da coluna d' água, proporcionado pela biomassa aérea desses vegetais. A reduzida concentração de oxigênio dissolvido também esteve relacionada ao sombreamento da coluna d'água, que inibe o desenvolvimento fitoplanctônico, e à decomposição da $\mathrm{MO}$ aderida ao sistema radicular de E. crassipes e P. stratiotes. De fato, Reddy \& DeBusk (1985) constataram que, em sistemas de tratamento de efluentes com macrófitas aquáticas com grandes lâminas foliares, como E. crassipes, $P$. stratiotes e Hydrocotyle umbellata, as concentrações de oxigênio dissolvido foram baixas (2,4-3,9 mg/L), especialmente em decorrência do sombreamento e da decomposição da matéria orgânica por organismos aeróbios. No sistema controle, a incidência dos raios solares sobre a superfície das unidades experimentais promoveu aumento dos valores de temperatura e favoreceu o desenvolvimento de algas filamentosas, que contribuíram para o aumento das concentrações de oxigênio e de $\mathrm{pH}$ no período diurno. Henry-Silva (2001), ao verificar a eficiência de macrófitas aquáticas no tratamento de piscicultura, também constatou grande desenvolvimento de fitoplâncton em tanques sem macrófitas, o que, conseqüentemente, promoveu aumento dos teores de oxigênio dissolvido na água durante o dia.

A remoção de nutrientes foi menor no sistema controle e semelhante entre os sistemas de tratamento com as macrófitas aquáticas (Figura 2). Nos tratamentos com macrófitas, foram constatados os maiores valores de remoção do fósforo total (41,9\% pelo controle; $71,6 \%$ por E. crassipes; $69,9 \%$ por $P$. stratiotes; $72,5 \%$ por $E$. crassipes $+P$. stratiotes e $72,1 \%$ por $P$. stratiotes $+E$. crassipes) e para turbidez (30,6\% pelo controle; $80,2 \%$ por E. crassipes; $75,2 \%$ por P. stratiotes; $79,8 \%$ por $E$. crassipes $+P$. stratiotes e $81,5 \%$ por $P$. stratiotes $+E$. crassipes). Entre as formas nitrogenadas, as maiores remoções foram verificadas para $\mathrm{N}$-nitrito $(14,8 \%$ pelo controle; $54,3 \%$ por $E$. crassipes; $54,5 \%$ por $P$. stratiotes; $51,1 \%$ por E. crassipes $+P$. stratiotes; e $52,7 \%$ por $P$. stratiotes $+E$. crassipes).

A eficiência dos sistemas com E. crassipes e P. stratiotes na redução da turbidez pode ser atribuída principalmente ao grande desenvolvimento radicular dessas duas espécies e à pouca profundidade dos tanques, que, provavelmente, criaram condições favoráveis para a adsorção e precipitação do material particulado em suspensão. Essa capacidade de retenção de material particulado é importante, pois os efluentes de aqüicultura geralmente são ricos em material orgânico, que pode provocar alterações nos ambientes aquáticos (Iwana, 1991; Henry-Silva \& Camargo, 2006).

A remoção de nutrientes, principalmente de fósforo, foi superior à observada em outros sistemas de tratamento de efluentes com maiores concentrações de nitrogênio e

Tabela 2 - Características limnológicas dos efluentes tratados pelos sistemas de tratamento com macrófitas aquáticas flutuantes

\begin{tabular}{|c|c|c|c|c|c|}
\hline Item & Controle & E. crassipes & P. stratiotes & $E+P$ & $P+E$ \\
\hline $\mathrm{pH}$ & $8,2 \pm 0,4(a)$ & $7,1 \pm 0,2(b)$ & $7,1 \pm 0,2(b)$ & $7,1 \pm 0,3(b)$ & $7,2 \pm 0,3(b)$ \\
\hline Condutividade $(\mathrm{mS} / \mathrm{cm})$ & $0,063 \pm 0,005(\mathrm{a})$ & $0,061 \pm 0,005(\mathrm{a})$ & $0,063 \pm 0,004(\mathrm{a})$ & $0,061 \pm 0,004(\mathrm{a})$ & $0,062 \pm 0,004(\mathrm{a})$ \\
\hline Turbidez (NTU) & $42 \pm 14,9(a)$ & $12 \pm 6,4(b)$ & $15,4 \pm 7,6(b)$ & $12,2 \pm 6,3(b)$ & $11,2 \pm 5,8(b)$ \\
\hline Fósforo total $(\mu \mathrm{g} / \mathrm{L})$ & $127,4 \pm 20,5(a)$ & $62,2 \pm 17,4(b)$ & $66,0 \pm 22,0(b)$ & $60,2 \pm 15,2(b)$ & $61,0 \pm 19,7(b)$ \\
\hline Fósforo dissolvido $(\mu \mathrm{g} / \mathrm{L})$ & $25,6 \pm 3,2(a)$ & $17,6 \pm 3,1(b)$ & $17,2 \pm 2,9(b)$ & $17,8 \pm 2,8(b)$ & $17,5 \pm 2,7(b)$ \\
\hline N-nitrito $(\mu \mathrm{g} / \mathrm{L})$ & $14,0 \pm 1,2(\mathrm{a})$ & $7,5 \pm 1,1(b)$ & $7,5 \pm 1,0(b)$ & $8,0 \pm 1,1(b)$ & $7,7 \pm 0,8(b)$ \\
\hline N-amoniacal $(\mu \mathrm{g} / \mathrm{L})$ & $16,0 \pm 1,0(\mathrm{a})$ & $11,5 \pm 0,9(b)$ & $11,1 \pm 0,9(b)$ & $11,2 \pm 0,9(b)$ & $11,5 \pm 0,9(b)$ \\
\hline
\end{tabular}

$\mathrm{E}+\mathrm{P}=$ Eichhornia crassipes + Pistia stratiotes. $\mathrm{P}+\mathrm{E}=$ Pistia stratiotes + Eichhornia crassipes. 
fósforo. Manfrinato (1989), utilizando E. crassipes para tratamento da água do Rio Piracicaba $(0,1-0,27 \mathrm{mg} / \mathrm{L}$ de fósforo total e 0,2-2,6 mg/L de $\mathrm{N}$-amoniacal), obteve reduções de até $47 \%$ nas concentrações de fósforo total. DeBusk et al. (1983) utilizaram a mesma espécie no tratamento de efluentes domésticos com altas concentrações de nutrientes $\left(0,7 \mathrm{mg} / \mathrm{L}\right.$ de $\mathrm{P}^{-} \mathrm{PO}_{4}$ e $3,2 \mathrm{mg} / \mathrm{L}$ de $\left.\mathrm{N}-\mathrm{NO}_{3}\right)$ e obtiveram remoções de 10\% de fósforo total. Redding et al. (1997), utilizando macrófitas aquáticas de diferentes grupos ecológicos no tratamento de efluentes de piscicultura com elevadas concentrações de $\mathrm{N}$-amoniacal $(0,2-4,3 \mathrm{mg} / \mathrm{L})$ e $\mathrm{N}$-nitrato $(1,2-11,7 \mathrm{mg} / \mathrm{L})$, constataram redução dessas formas nitrogenadas, respectivamente, de 4,3 e 3,3\% por Azolla filiculoides (flutuante), 8,2 e 5,9\% por Elodea nuttalli (submersa) e 10,7 e 15,4\% por Rorippa nasturtiumaquaticum (emersa).

Esses resultados indicam que a eficiência desses sistemas de tratamento depende das concentrações de nutrientes nos efluentes e das espécies de macrófitas aquáticas utilizadas. É importante salientar que os processos de remoção de nutrientes nestes sistemas ocorrem não somente pela absorção direta pelas macrófitas aquáticas, mas também por uma combinação de mecanismos físicos, biológicos e químicos, como sedimentação, absorção pelo perifíton e transformação do nitrogênio por bactérias.

Apesar da eficiência das macrófitas aquáticas na remoção de nutrientes e na redução das cargas de matéria orgânica do efluente de carcinicultura, ressalta-se a neces- sidade de oxigenação desses efluentes tratados, especialmente se forem reutilizados em atividades de aqüicultura. Em sistemas aqüícolas com alta densidade de cultivo, baixas taxas de troca de água, baixo $\mathrm{pH}$ e reduzidas concentrações de oxigênio dissolvido, é provável que o dióxido de carbono dissolvido se acumule em níveis que possam criar condições tóxicas para os organismos heterotróficos.

Uma alternativa para incrementar as concentrações de oxigênio dissolvido é a utilização de sistemas de tratamento com espécies de macrófitas aquáticas submersas e algas. Tripathi \& Shukla (1990) também verificaram redução nas concentrações de oxigênio dissolvido após os efluentes urbanos serem tratados por um sistema com E. crassipes (0,64-1,4 mg/L). Para aumentar os valores de oxigênio, foi instalado um sistema com Chlorella vulgares, Scenedesmus quadricauda, Microcystis aeruginosa e Euglena viridis. Essas algas, por meio de suas atividades fotossintéticas, proporcionaram considerável aumento das concentrações de oxigênio dissolvido (3-6-14,5 mg/L). A macrófita aquática Egeria densa, apesar da menor eficiência na remoção de nutrientes, também proporciona condições para o aumento da oxigenação dos efluentes tratados (Reddy \& DeBusk, 1985).

O tempo de residência do efluente nos sistemas de tratamento é outro aspecto que deve ser considerado, uma vez que a alta vazão geralmente dificulta os processos de absorção de nutrientes e de sedimentação de material particulado. De fato, estudo realizado com E. crassipes

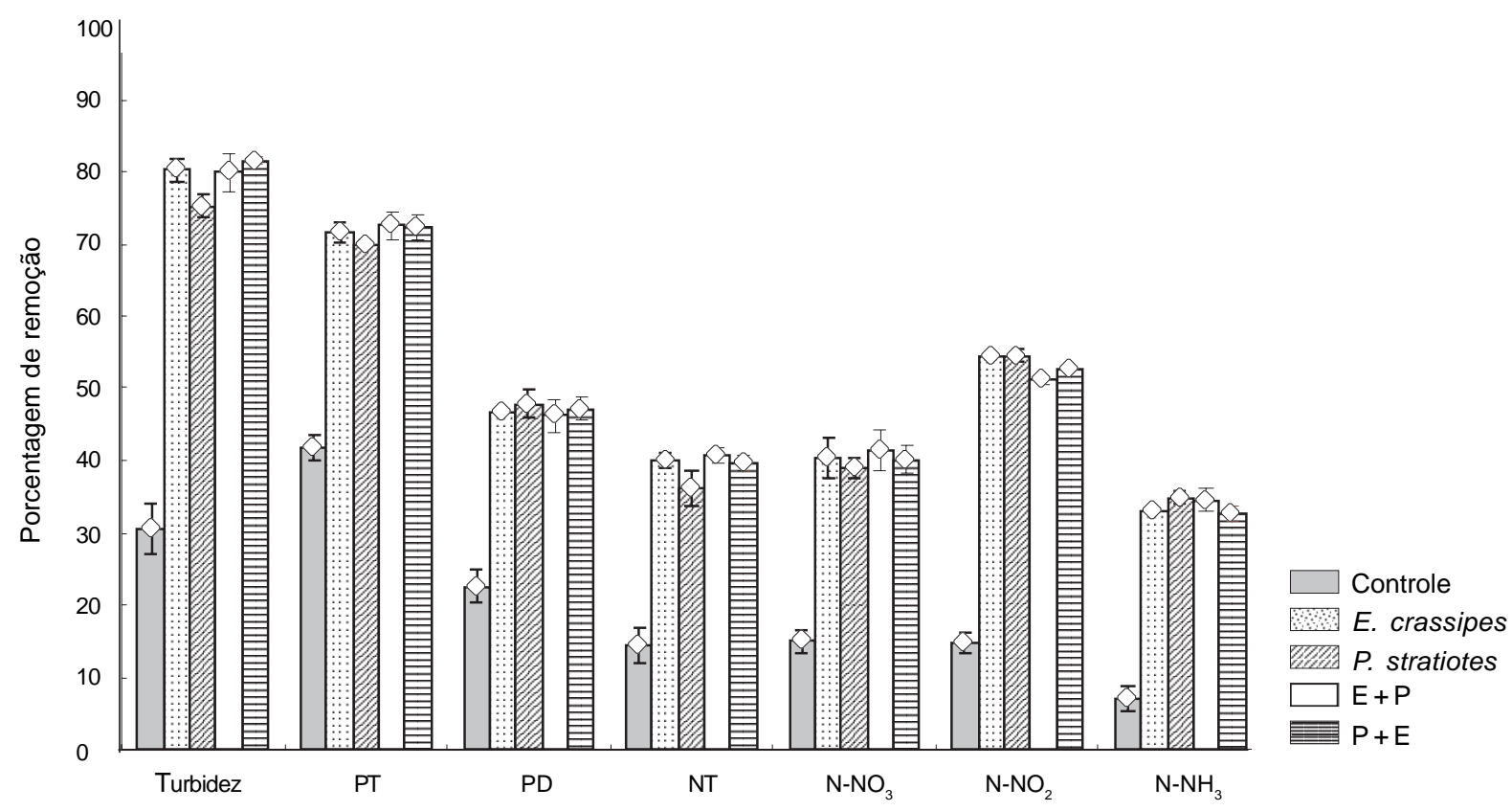

Figura 2 - Redução da turbidez e remoção de fósforo total (PT), fósforo dissolvido (PD), nitrogênio total (NT), N-nitrato (N-NO 3 ), N-nitrito $\left(\mathrm{N}-\mathrm{NO}_{2}\right)$ e $\mathrm{N}$-amoniacal $\left(\mathrm{N}-\mathrm{NH}_{3}\right)$ pelos sistemas de tratamento de efluentes. $\mathrm{E}+\mathrm{P}=$ Eichhornia crassipes e Pistia stratiotes e $\mathrm{P}+\mathrm{E}=$ Pistia stratiotes e Eichhornia crassipes. 
em um sistema de tratamento de efluentes de aqüicultura com elevada vazão, comprovou acréscimo dos valores de $\mathrm{N}$-nitrato, fósforo total e P-ortofosfato no efluente tratado (Sipaúba-Tavares et al., 2001). Pesquisas em alagados artificiais com macrófitas aquáticas emersas comprovaram correlação positiva entre a eficiência na remoção de nutrientes e o tempo de residência do efluente no alagado (Sakadevan \& Bavor, 1999). Neste trabalho, a vazão utilizada nos sistemas de tratamento (17 horas) foi suficiente para a remoção da maior parte do fósforo e da turbidez nos efluentes de carcinicultura.

Os sistemas de tratamento com macrófitas aquáticas apresentaram eficiências similares na redução dos valores de nutrientes e de turbidez. No entanto, o ganho de biomassa em E. crassipes foi superior ao de P. stratiotes (Figura 3). Além disso, a maximização da retirada de nitrogênio e fósforo depende da remoção contínua das macrófitas, uma vez que essas plantas estocam nutrientes apenas por um curto período detempo. Assim, a utilização de E. crassipes em sistemas de tratamento de efluentes de carcinicultura é mais indicada quando há aproveitamento da biomassa vegetal. Caso contrário, é recomendável a utilização de $P$. stratiotes, em razão da maior facilidade na remoção da biomassa vegetal excedente.

Os resultados comprovaram efetiva capacidade das macrófitas aquáticas flutuantes em remover matéria orgânica particulada e nutrientes dos efluentes de carcinicultura. Considerando que a qualidade do efluente gerado pelas atividades de aqüicultura não deve diferir da qualidade da água utilizada nas criações (Mires, 1995), pode-se afirmar que os sistemas de tratamento com E. crassipes e $P$. stratiotes atingiram grande eficiência, pois os teores de nitrogênio e fósforo nos efluentes tratados foram menores que na água de abastecimento do viveiro de camarões. Portanto, após o tratamento dos efluentes com macrófitas aquáticas, é possível reutilizar esses efluentes nas atividades de carcinicultura, ou mes mo, lançá-los nos ambientes aquáticos naturais minimizando os impactos relacionados à eutrofização artificial.
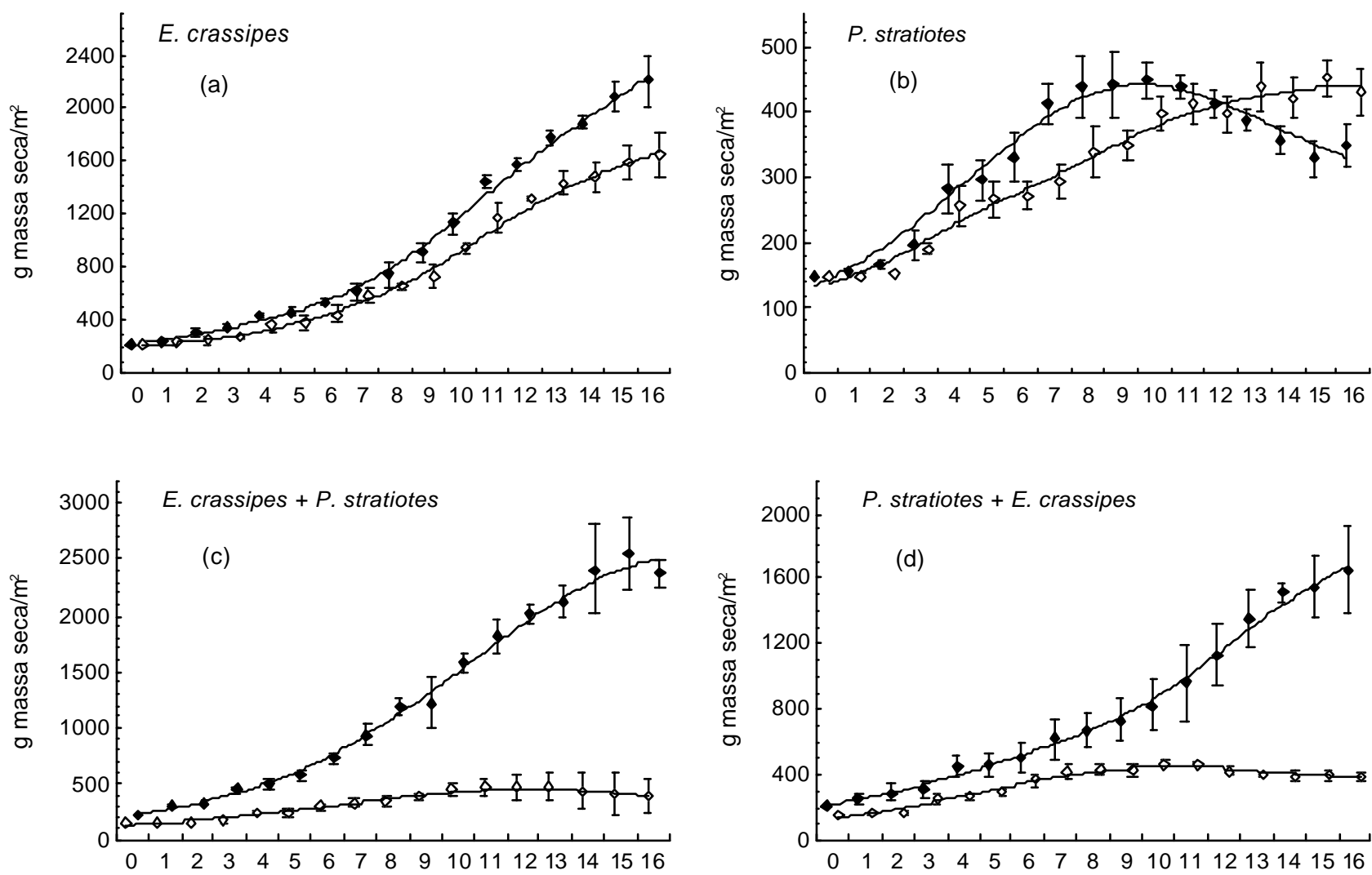

Semana

Figura 3 - Crescimento das macrófitas aquáticas nos sistemas de tratamento E. crassipes (a); P. stratiotes (b); E. crassipes + $P$. stratiotes $(\mathrm{c}) ; P$. stratiotes $+E$. crassipes $(\mathrm{d})$ na metade inicial $(\diamond)$ e na metade final $(\diamond)$ das unidades experimentais. 


\section{Conclusões}

As macrófitas aquáticas Eichhornia crassipes e Pistia stratiotes foram eficientes na remoção de nitrogênio, fósforo e turbidez dos efluentes de carcinicultura e não diferiram significativamente quanto à remoção de nutrientes, apesar do maior ganho de biomassa de E. crassipes.

\section{Agradecimento}

Ao Prof. Dr. Wagner Cotroni Valenti, pela disponibilização da infra-estrutura do setor de Carcinicultura (UNESP Jaboticabal) e pelas valiosas sugestões; aos técnicos Carlos Fernando Sanches, Valdecir Fernandes de Lima e José Roberto Polachini, pelo auxílio nos trabalhos de campo e de laboratório; e à FAPESP, pelo suporte financeiro (Processo: 02/04131-8).

\section{Literatura Citada}

ASSAD, L.T.; BURSZTYN, M. Aqüicultura sustentável. In: VALENTI, W.C.; POLI, C.R.; PEREIRA, J.A. et al. (Eds.) Aqüicultura no Brasil, bases para um desenvolvimento sustentável. Brasília: CNPq/Ministério da Ciência e Tecnologia, 2000. p.33-72.

BACCARIN, A.E.; CAMARGO, A.F.M. Characterization and evaluation of the impact of feed management on the effluents of Nile tilapia (Oreochromis niloticus) culture. Brazilian Archives of Biology and Technology, v.48, p.81-90, 2005.

BEARDMORE, J.A.; MAIR, G.C.; LEWIS, R.I. Biodiversity in aquatic systems in relation to aquaculture. Aquaculture Research, v.28, p.829-839, 1997.

BOYD, C. Guidelines for aquaculture effluent management at the farm-level. Aquaculture, v.226, p.101-112, 2003

CAstagnolli, N. Piscicultura de água doce. Jaboticabal: FUNESP/FCAVJ/UNESP, 1992. 189p.

CAVAlCANTI, L.B. Histórico. In: VAlEnTI, W.C. (Ed.). Carcinicultura de água doce: tecnologia para produção de camarões. Brasília: Instituto Brasileiro do Meio Ambiente e dos Recursos Naturais Renováveis, 1998. p.17-20.

DEBUSK, T.A.; WILLIAMS, L.D.; RYTHER, J.H. Removal of nitrogen and phosphorus from waste in a waterhyacinth-based treatment system. Journal of Environmental Quality, v.12, n.2, p.257-262, 1983.

ENNABILI, A.; ATER, M.; RADOUX, M. Biomass production and NPK retention in macrophytes from wetlands of the Tingitan Peninsula. Aquatic Botany, v.62, p.45-56, 1998.

GOLTERMAN, H.L.; CLYNO, R.S.; OHNSTAD, M.A.M. Methods for chemical analysis of fresh water. Boston: Blackwell, 1978, 214p.

GREENWAY, M. The role of constructed wetlands in secondary effluent treatment and water reuse in subtropical and arid Australia. Ecological Engineering, v.25, p.501-509, 2005.

HADAD, H.R.; MAINE, M.A.; BONETTO, C.A. Macrophyte growth in a pilot-scale constructed wetland for industrial wastewater treatment. Chemosphere, v.63, p.1744-1753, 2006.

HENRY-SILVA, G.G.; CAMARGO, A.F.M.; PEZZATO, L.E. Digestibilidade aparente de macrófitas aquáticas pela tilápia do nilo (Oreochromis niloticus) e qualidade da água em relação às concentrações de nutrientes. Revista Brasileira de Zootecnia, v.35, n.3, p.642-647, 2006.
HENRY-SILVA, G.G. Utilização de macrófitas aquáticas flutuantes (Eichhornia crassipes, Pistia stratiotes e Salvinia molesta) no tratamento de efluentes de piscicultura e possibilidades de aproveitamento da biomassa vegetal. Jaboticabal: Universidade Estadual Paulista, 2001. 56p. Dissertação (Mestrado em Aqüicultura) - Universidade Estadual Paulista, 2001

HENRY-SILVA, G.G. Tratamento de efluentes de carcinicultura por macrófitas aquáticas, interações ecológicas e valor nutritivo da biomassa vegetal para tilápia do Nilo. Jaboticabal: Universidade Estadual Paulista, 2005. 132p. Tese (Doutorado em Aqüicultura) - Universidade Estadual Paulista, 2005.

HENRY-SILVA, G.G.; CAMARGO, A.F.M. Impacto do lançamento de efluentes urbanos sobre alguns ecossistemas aquáticos do município de Rio Claro (SP). Revista Ciências Biológicas e do Ambiente, v.2, p.317-330, 2000.

HENRY-SILVA, G.G.; CAMARGO, A.F.M. Efficiency of aquatic macrophytes to treat Nile tilapia pond effluents. Scientia Agrícola, v.63, p.433-438, 2006.

IWAMA, K.G. Interactions between aquaculture and the environment. Critical Reviews in Environmental Control, v.21, n.2, p.177-216, 1991 .

KOROLEFF, F. Determination of nutrients. In: GRASSHOFF, K. (Ed.) Methods of seawater analysis. Wwinhein: Verlog. Chemic., 1976. p.117-181.

LIN, Y.F.; JING, S.R.; LEE, D.Y. et al. Performance of a constructed wetland treating intensive shrimp aquaculture wastewater under high hydraulic loading rate. Aquaculture, v.134, p.411-421, 2005.

MACKERETH, F.I.F.; HERON, J.; TALLING, J.F. Water analysis: some revised methods for limnologist. London: Freswater Biological Association, 1978. 121p.

MANFRINATO, E.S. Avaliação do método edafo-fitodepuração para tratamento preliminar de águas. Piracicaba: Escola Superior de Agricultura Luiz de Queiroz, 1989. 98p. Dissertação (Mestrado em Agronomia) - Escola Superior de Agricultura Luiz de Queiroz, 1989.

MIDLEN, A.; REDDING, T. Environmental management for aquaculture. Netherlands: Kluwer Academic Publishers, 1998. $223 p$.

MIRES, D. Aquaculture and the aquatic environment: mutual impact and preventive management. The Israeli Journal of Aquaculture, v.47, p.163-172, 1995.

NACA/FAO. Aquaculture development beyond 2000: the Bangkok declaration and strategy. In: CONFERENCE ON AQUACULTURE IN THE THIRD MILlENNIUM, 2000, Bangkok. Proceedings... Bangkok: NACA, Bangkok \& FAO, 2000. 27p.

OSTRENSKY, A.; BORGHETTI, J.R.; PEDINI, M. Situação atual da aqüicultura brasileira e mundial. In: VALENTI, W.C; POLI, C.R.; PEREIRA, J.A. et al. (Eds.) Aqüicultura no Brasil, bases para um desenvolvimento sustentável. Brasília: CNPQ/Ministério da Ciência e Tecnologia, 2000. p.353-382.

PILARSKI, F.; TOMAZELLI JR., O; CASATA, J.C. et al. Consórcio suíno-peixe: aspectos ambientais e qualidade do pescado. Revista Brasileira Zootecnia, v.33, n.2, 267-276, 2004.

REDDING, T.; TODD, S.; MIDLEN, A. The treatment of aquaculture wastewater - A botanical approach. Journal of Environmental Management, v.50, p.283-299, 1997.

REDDY, K.R.; DEBUSK, T.A. Nutrient removal potential of selected aquatic macrophytes. Journal of Environmental Quality, v.14, n.4, p.459-462, 1985.

SAKADEVAN, K.; BAVOR, H.J. Nutrient removal mechanisms in constructed wetlands and sustainable water management. Water Science Technology, v.40, n.2, p.121-128, 1999.

SIPAÚBA-TAVARES, L.H.; FÁVERO, E.G.P.; BRAGA, F.M. Utilização de biofiltros de macrófitas como forma de minimizar os impactos causados pela aqüicultura. In: SEMINÁRIO INTERNACIONAL DA REPRESA DO LOBO-BROA, 1., 2001, São Carlos. Anais... São Carlos: 2001. p.151. 
SIPAÚBA-TAVARES, L.H.; FÁVERO, E.G.P.; BRAGA, F.M.S. Utilization of macrophyte biofilter in effluent from aquaculture: I. Floatin plant. Brazilian Journal of Biology, v.62, n.3, p.1-11, 2002.

TRIPATHI, A.; SHUKLA, S.C. Biological treatment of wastewater by selected aquatic plants. Environmental Quality, v.69, p.69-78, 1990 .

TRUE, B; JOHNSON, W.; CHEN, S. Reducing phosphorus discharge flow through aquaculture I: facility and effluent characterization. Aquacultural Engineering, v.32, p.129-144, 2004.
VALENTI, W.C. Aquaculture for sustainable development. In: VALENTI, W.C.; POLI, C.R.; PEREIRA, J.A.; BORGHETTI, J.R. (Eds.) Aqüicultura no Brasil, bases para um desenvolvimento sustentável. Brasília: $\mathrm{CNPQ} /$ Ministério da Ciência e Tecnologia, 2000. p.17-24.

WANG, J. Managing shrimp pond water to reduce discharge problems. Aquaculture Engineering, v.9, p.134-142, 1990

ZANIBONI FILHO, E.; BARBOSA, N.D.C.; GONCALVES, S.M.R Caracterização e tratamento do efluente das estações de piscicultura. Acta Scientiarum, v.19, n.2, p.537-548, 1997. 